\title{
Caractérisation des houles extrêmes pour l'industrie offshore Apport des données satellitaires
}

\author{
Caracterisation of extreme swells for offshore industry \\ Contribution of satellite data
}

par Michel Olagnon

Ifremer

Extreme wave design conditions are of high concern to the offshore industry, especially since many developments are planned in poorly documented areas from a climatological point of view, and since the safety requirements are most stringent. With the availability of a whole decade of satellite observations of sea states, their integration into the state of the art and the remaining difficulties are discussed here. An example is taken with the Iroise Sea, and perspectives are envisionned.

\section{I $\square$ LE CONTEXTE}

L'industrie offshore, qui est sur le point de publier sous forme de normes internationales ce qui n'était jusqu'à présent que des recommandations complétées par des réglementations nationales, utilise pour le dimensionnement des ouvrages une approche fondée sur deux grands principes : la confrontation probabiliste des chargements et des résistances, et la détermination des niveaux de fiabilité à atteindre en fonction des conséquences encourues. Typiquement, ces niveaux vont d'un risque de $10^{-2}$ annuel pour un ouvrage inhabité sans risque environnemental à $10^{-4}$ annuel pour un ouvrage habité ou susceptible de causer un risque environnemental. Ils sont conçus pour que les risques liés aux erreurs de conception soient inférieurs d'au moins un ordre de grandeur à ceux résultant des erreurs humaines.

La nature des nouveaux concepts utilisés pour l'exploration et la production des hydrocarbures en mer fait qu'une telle procédure est particulièrement gourmande en caractérisations des états de mer. En effet, on ne peut plus se satisfaire d'estimer la hauteur qui n'est dépassée qu'avec une probabilité infime donnée telle la vague centenale ou dix-millénale, comme on pouvait le faire pour la plupart des structures fixes de type treillis. Il est nécessaire d'envisager toutes les situations, c'est-à-dire les combinaisons de paramètres excitateurs et de réponses dynamiques, qui peuvent amener, parfois par le biais d'un scénario de ruine complexe, aux conséquences indésirables dont on souhaite réduire le risque d'occurrence. Ainsi, la condition va dépendre de la structure considérée et de ses modes de réponse aux sollicitations des vagues.

Pour une structure fixe posée sur le fond, on s'intéressera à la hauteur maximale des vagues individuelles (qui, déjà, n'apparaît pas forcément lors de l'état de mer de plus forte hauteur significative), à la période ou à la cambrure associée qui régit les forces sur les membrures, mais aussi à l'élévation maximale de la crête par rapport au niveau moyen (crête non-linéaire + marée + surcote) et à la direction de provenance de cette vague si la structure peut être orientée pour opposer une plus grande résistance dans une certaine direction. Ces éléments restent peu éloignés de ceux qui ont servi par le passé au dimensionnement d'autres structures fixes. Par contre, pour un flotteur allongé ancré, comme par exemple une barge, la forme spectrale risque d'être déterminante et de conduire à des sollicitations maximales pour des hauteurs de vagues seulement sub-extrêmes, ou même encore moindres, mais associées à des périodes spécifiques, du fait de résonnances. De manière similaire, l'étalement directionnel des vagues incidentes peut conduire à des réponses transverses dangereuses d'un flotteur qui s'oriente face à la direction dominante des vents et des courants tout autant que des vagues.

Par exemple, le FPSO (Floating Production Storage and Offloading) de Foinhaven s'est trouvé en situation extrême peu après son installation simplement du fait de l'arrivée soudaine d'une forte houle de travers alors que les hauteurs totales de vagues restaient en deça des valeurs extrêmes prévues sur la zone. De même, dans la nuit du 9 novembre 98 , et par des conditions de mer de $14 \mathrm{~m}$ de hauteur significative et 15-16 secondes de période, une partie de la proue du FPSO de Schiehallion, à 20 mètres au dessus de la flottaison fut enfoncée de 25 centimètres.

Les aspects liés à l'opérationnalité et à la fatigue conduisent également à donner une autre acceptation au terme " extrêmes ", ou, au minimum, aux conditions qui vont servir au dimensionnement. Les risques peuvent en effet être plus élevés en raison d'opérations comme le chargement d'un pétrolier navette dans des conditions pouvant conduire à la collision avec le FPSO ou à la rupture des lignes de liaison, 


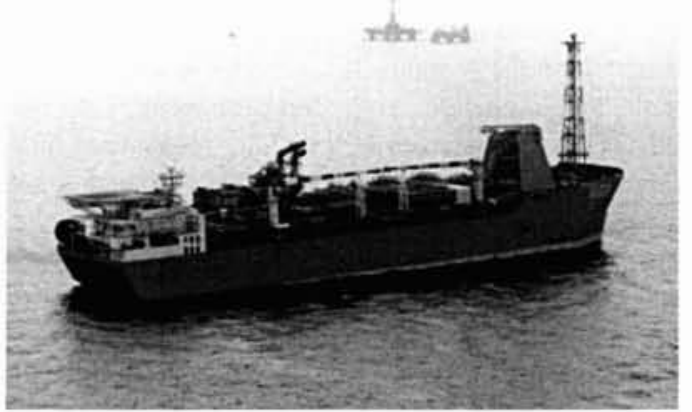

Figure 1.

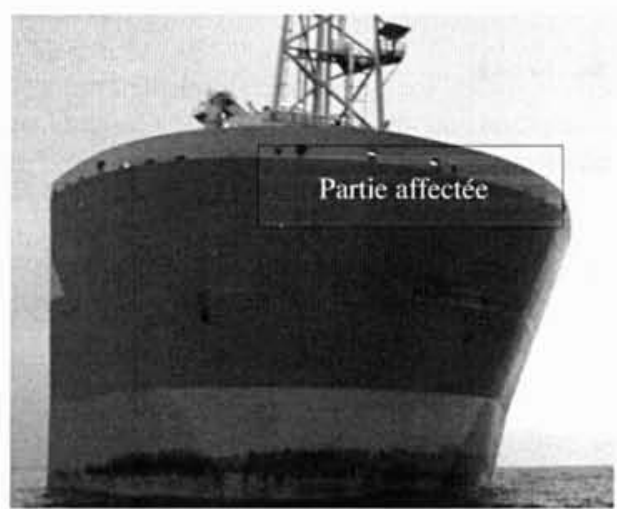

Figure 2.

que dans des conditions extrêmes où des dispositions de type « survie " ont pu être prises. Les conditions extrêmes sont alors, là encore, des conditions où la hauteur des vagues est relativement loin du maximum possible.

\section{II $\square$ LES CLIMATOLOGIES OPÉRATIONNELLES OFFSHORE}

\section{II.1 Nature}

Une caractérisation complète des états de mer pour l'offshore comprend donc, en général, outre les hauteurs significative et maximale des vagues, les périodes associées, les directions principales, et éventuellement la largeur de bande spectrale et l'étalement directionnel, de préférence pour chacun des principaux pics spectraux, c'est-à-dire des trains de vagues qui composent l'état de mer. Lorsque c'est possible, il convient d'ajouter à cette description le vent et le courant qui pourraient être observés conjointement. Cette caractérisation doit être disponible pour l'ensemble des situations pouvant conduire à la probabilité donnée de dépassement de la résistance de l'ouvrage aux sollicitations. Il faut donc évaluer les probabilités conjointes des différents paramètres pour toute une région de l'espace de leurs combinaisons, soit pour différents cas de charge, suffisamment différents.

Il est évident qu'avec des exigences aussi élevées, la constitution des climatologies requises représente une tâche considérable. Historiquement, les observations de navires et les quelques mesures in situ disponibles ont représenté les sources de données les plus conséquentes, pour ne pas dire uniques. La dernière décennie a vu une prise d'importance de deux nouvelles sources, censées pallier les défauts, zones mal couvertes et biais, des précédentes : le hindcast (rétrosimulation) et la mesure satellitaire.

Pour les opérateurs offshore, le hindcast présente de nombreux avantages :

- La possibilité de construire en un temps compatible avec les temps de développement des champs pétroliers une base de données d'une durée - typiquement 40 ou 50 ans -acceptable pour l'extrapolation aux extrêmes sur la durée de vie des ouvrages considérés.

— La génération de spectres directionnels complets, en tout point de la grille où on le désire.

- La calibration par rapport aux mesures disponibles qui peut se faire avec une colocalisation parfaite en temps et en espace.

- Les historiques complets, sans manques, que fournit le hindcast et qui peuvent être utilisés pour des simulations de type Monte-Carlo en vue par exemple des opérations marines, dont on a vu plus haut l'importance pour le dimensionnement. — La possibilité de partager les coûts élevés de ces études entre l'ensemble des opérateurs actifs dans une région, en raison de la taille des bassins océaniques traités.

Par contre, un certain nombre de problèmes demeurent, particulièrement ceux liés à la nature essentiellement empirique des ajustements de calibration et de validation, et à la dépendance de la qualité des résultats envers les choix de l'opérateur humain du programme informatique. D'autre part, par leur nature même, les extrêmes sont rares tant dans la simulation que dans la nature, et les calibrations ne peuvent pas atteindre le niveau élevé de fiabilité cherché pour des événements aussi rares.

\section{- II.2 Contribution des données satellitaires}

Au bout de dix années de collecte qui ont pourtant largement effacé les questions de densité de l'échantillonnage qu'on se posait à leurs débuts, les données satellitaires restent paradoxalement très modérément utilisées dans l'industrie offshore, et presque toujours en complément à d'autres sources de données. On peut penser que trop d'efforts ont porté sur la promotion des données satellitaires en elles-mêmes, et pas assez sur la résolution, en combinaison avec d'autres sources, de deux problèmes fondamentaux :

- L'évaluation d'historiques et de distributions au point fixe à partir de mesures quasi-instantanées à intervalles éloignés tout au long de traces géographiquement étendues; et les modifications à apporter en conséquence aux procédures de calcul d'extrêmes.

- Le choix des paramètres à associer, pour les quantités que le satellite ne peut mesurer, à celles qui ont pu l'être (périodes, formes spectrales, directions).

Mis à part pour certaines zones tropicales où les extrêmes sont déterminés par un petit nombre de cyclones d'une extension géographique très restreinte, et que l'observation satellitaire a toutes les chances de "manquer ", les données satellitaires permettent pourtant, au large, l'estimation la plus précise et la plus fiable de la queue de la distribution des hauteurs significatives. C'est particulièrement significatif pour les régions qui ne disposent ni d'une longue histoire ni d'une forte densité d'observations météorologiques permettant de calibrer les hindcasts ou d'extrapoler des mesures in situ. La formalisation de nouvelles procédures adaptées de passage 
aux extrêmes ne présente pas de difficulté insurmontable, mais reste insuffisante, et n'est surtout malheureusement pas encore traduite dans les règlements, les recommandations et les normes. De plus, des résultats de calculs hâtifs ont pu montrer des discordances avec les attentes des spécialistes, et jeter des doutes sur la qualité des mesures satellitaires et de leur calibration.

\section{- II.3 Illustration concrète}

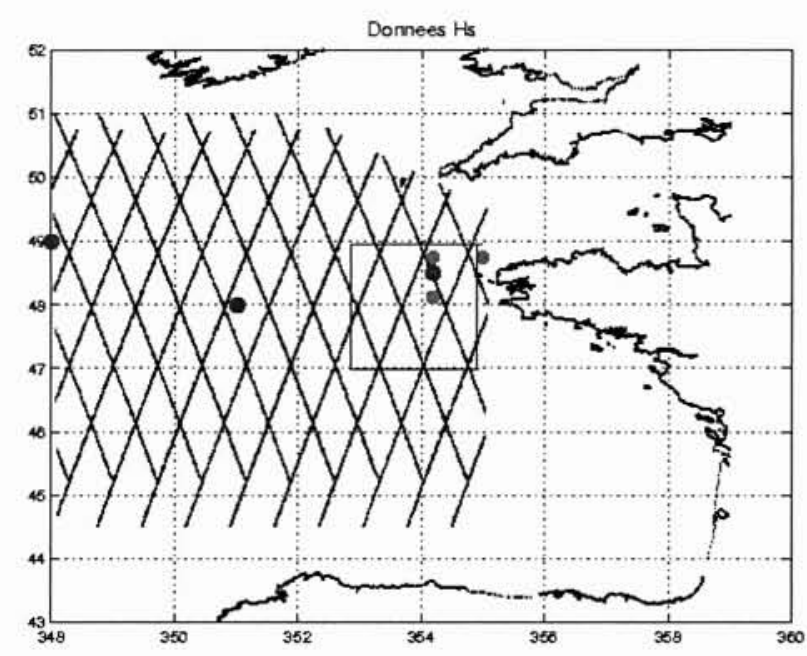

Figure 3.

A titre d'exemple, examinons le cas de la mer d'Iroise. La figure 3 montre les sources de données qui pourraient être utilisées pour bâtir une climatologie d'extrêmes à l'entrée du rail d'Ouessant :

- Mesures ponctuelles des bouées météo, assez éloignées vers l'ouest, et de la bouée Béatrice au point d'intérêt.

- Trois des points de grille les plus proches du hindcast AES-40.

— Traces de mesure de la hauteur significative par le satellite ERS.

Ce site est particulièrement favorisé du point de vue estimation de la climatologie en raison de la présence de la bouée Béatrice, qui fournit des spectres directionnels complets de l'état de mer. Toutefois même dans ce cas, les évolutions du capteur ne garantissent pas l'homogénété des huit années de mesure disponibles et cette durée reste faible pour rechercher des «points de dimensionnement " dont la probabilité d'occurrence annuelle doit être de $10^{-2}$, et encore moins pour $10^{-4}$.

Le hindcast est le seul dont la durée soit raisonnable par rapport à de tels objectifs. Il présente cependant l'inconvénient de requérir une calibration des formes spectrales et des valeurs rares. Dans la zone considérée, cette calibration peut se faire a posteriori sur les mesures de Béatrice, même si en toute rigueur, ces mesures devraient être prises en compte au moment de la simulation numérique dans la réanalyse des tempêtes. Le travail n'en est pas moins conséquent. Il serait quasi-impossible en un lieu où on ne disposerait pas de mesures in situ directionnelles.

L'observation satellitaire, rappelons-le, met en œuvre trois principaux types de capteurs d'intérêt pour la climatologie des états de mer [1]
— L'altimètre. Ce capteur mesure la rugosité de la surface libre, qui est retournée comme une perturbation de la mesure de l'altitude précise du satellite par rapport à la surface. On obtient ainsi la hauteur significative des vagues sur une tache d'environ $7 \mathrm{~km}$ par 4 , toutes les secondes le long de la trace à la verticale du satellite, et la force du vent. Une pseudopériode peut également en être déduite moyennant quelques hypothèses assez réductrices, dont la validité reste à démontrer en dehors des conditions les plus courantes.

- Le radar à synthèse d'ouverture (SAR). Ce capteur produit des images radar de la surface libre, qui donnent accès à des spectres directionnels des vagues. Il est malheureusement limité par de nombreux facteurs : une résolution qui oblitère les vagues de longueur d'onde inférieure à une quarantaine de mètres, des secteurs quasi-aveugles dans la direction de déplacement du satellite, une dépendance envers l'existence d'un vent local pour mesurer quoi que ce soit, et une fonction de transfert non-linéaire et non-explicitement inversible.

- Le diffusiomètre. Ce capteur permet de déterminer le vent, force et direction, sur une "fauchée " de plusieurs centaines de kilomètres soit sur le côté, soit de part et d'autre de l'orbite. Même lorsqu'on s'intéresse aux vagues, ces champs de vent peuvent avoir une grande utilité pour pallier les défauts et les manques des précédents capteurs.

On retrouve ces différents capteurs sur la figure 4, tirée de l'ouvrage cité plus haut [1]. Le long de la trace du satellite, l'altimètre fournit la mesure de la hauteur significative et de la force du vent, représentées sur la vignette à gauche de cette trace. On note que les données requièrent une validation attentive, le passage sur des terres émergées se traduisant par des valeurs aberrantes, mais qui pourraient dans le cas d'îles de faible surface apparaître comme des extrêmes crédibles. A la droite de cette trace montante, on retrouve le champ de vent mesuré par le diffusiomètre, avec sa force et sa direction. Le sens n'est en fait obtenu qu'après un lever de doute à partir de sorties des modèles météo, et là encore, des erreurs peuvent se glisser, à proximité des fronts météorologiques et des centres de dépressions, quant au sens réel. Dans ce champ de vent, tous les deux cents kilomètres environ, le radar à synthèse d'ouverture mesure une imagette qui permet d'estimer un spectre directionnel. Ce mode vague du radar à synthèse d'ouverture n'en demeure pas moins d'un traitement délicat. La rose sur la droite de la figure montre le type de spectre directionnel qui peut en être déduit.

Les mesures satellitaires représentent une durée de dix ans, mais sont effectuées avec un échantillonnage très lâche et de nombreuses tempêtes sont manquées si on se limite à un carré restreint autour du point d'intérêt; la question de l'identité climatologique se pose pour agrandir cette zone. Il faut d'autre part établir quelle est la durée en un point équivalente à l'ensemble de mesures géographiquement dispersées retenues.

Cet aspect donne souvent lieu à des approximations injustifiées. On ne peut, en effet, compter qu'on dispose de dix années de mesure satellitaire dans une zone donnée simplement parce que les satellites ERS ont été maintenant en opération pendant dix ans. Il convient d'une part de comparer le nombre des vagues individuelles intervenant dans la climatologie satellitaire avec le nombre qui en aurait été observé au point fixe pendant cette durée de dix ans, et d'autre part de vérifier que les tempêtes retenues sont météorologiquement semblables et en nombre comparable à celles qui sont passées en un point fixe pendant cette même durée. Tâche délicate 


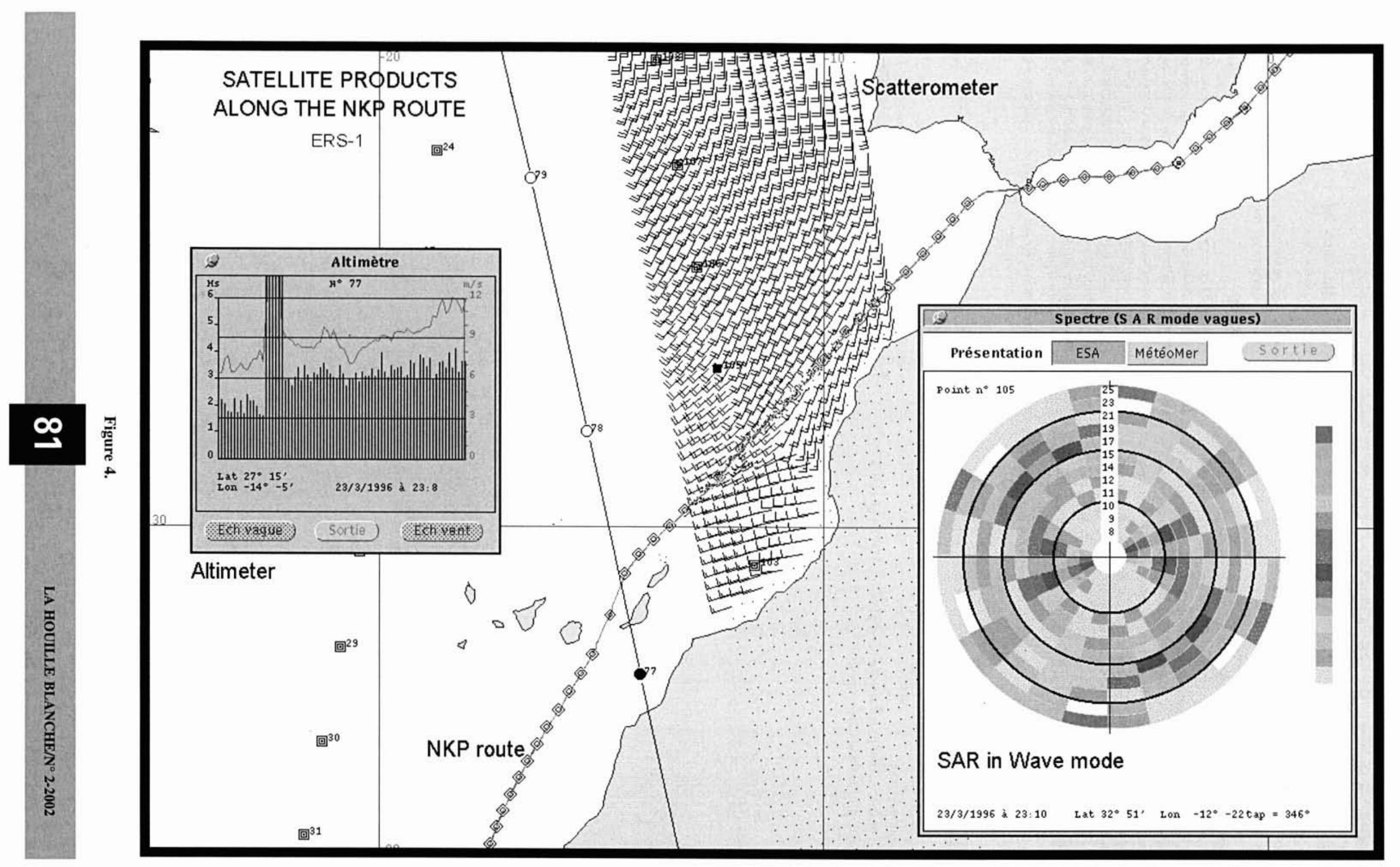


lorsqu'on veut recourir aux mesures satellitaires justement parce qu'on ne dispose pas de mesures in situ et qu'on n'a qu'une confiance limitée dans le hindcast en conditions de tempête !

Enfin, le problème d'associer aux hauteurs significatives mesurées les autres paramètres, période, direction, largeur spectrale en fréquence et en direction, nécessaires au calcul de la réponse d'un ouvrage, reste entier. Il peut certes être contourné par le recours au hindcast et/ou aux estimations qu'on peut faire avec la pseudo-période dérivée de l'altimètre, le SAR ou le diffusiomêtre, mais seulement pour des conditions usuelles. L'extrapolation aux extrêmes reste hasardeuse, pour ne pas dire impossible si le paramètre dimensionnant est par exemple plus la période que la hauteur de la houle comme c'est le cas pour certains ouvrages ancrés.

On voit donc qu'en l'absence de mesures in situ, les seules mesures satellitaires ne donneront qu'une description partielle de la climatologie, et qu'il faudra le plus souvent les compléter par le hindcast ou une campagne d'instrumentation, dont, il est vrai, on pourra probablement réduire la durée et le coût.

\section{III $\square$ CONCLUSION}

Les travaux en cours devraient aboutir à des méthodes reconnues pour :

- constituer des bases de données plus semblables à celles issues d'historiques en un point ;
— extrapoler aux extrêmes à utiliser pour la conception des ouvrages ;

— associer aux paramètres qui sont mesurés des valeurs statistiquement réalistes pour ceux qui ne le sont pas.

Ces aspects devraient bénéficier également aux données issues d'autres sources, et montrer la nécessité d'intégrer toutes les sources.

Enfin, on ne peut passer sous silence la question du coût. Jusqu'à présent, la mise des données satellitaires à disposition de l'industrie s'est faite pour des coûts marginaux, le financement de la mesure étant assuré par des grands programmes essentiellement scientifiques. Les impératifs de sécurité citoyenne que leur utilisation dans le dimensionnement permet de satisfaire justifient la poursuite de cette politique, mais on peut toujours craindre qu'une volonté de faire payer à l'industriel la plus grande partie du coût de la mesure ne se fasse jour, et n'hypothèque à jamais l'avenir des données satellitaires pour les ouvrages en mer.

\section{BIBLIOGRAPHIE}

[1] Aage Ch., Allan T., Carter D.J.T., Lindgren G., Ollagnon M. \{lromanl\} (1999). - Oceans from Space - A textbook for Offshore Engineers and Naval Architects. Ifremer, Repères Océans 16, 176 pp., ISBN 2-84433-010-X. 